\title{
Polycomb protein EZH2 regulates E2F1-dependent apoptosis through epigenetically modulating Bim expression
}

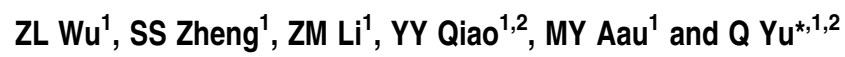

Deregulation of the $\mathrm{PRB} / \mathrm{E} 2 \mathrm{~F}$ pathway, which occurs frequently in human malignancy, is often associated with inappropriate proliferation and/or apoptosis. While the role of E2F1 in apoptosis induction has been well-established, it remains unclear how this pro-apoptotic activity is regulated in cancer. Here we describe EZH2, an oncogenic polycomb histone methyltransferase and an E2F1 target, as an important regulator of E2F1-dependent apoptosis. We show that E2F1 induces EZH2 expression, which in turn antagonizes the induction of E2F1 pro-apoptotic target Bim expression. RNAi-mediated gene depletion of EZH2 enhances E2F1-dependent Bim expression, thereby promoting the pro-apoptotic activity of E2F1. Hence, the concomitant induction of EZH2 and Bim by E2F1 constitutes a fail-safe mechanism to allow tumor cells with aberrant E2F1 activity to evade apoptosis. These findings reveal a novel mechanism by which the apoptotic activity of E2F1 is restrained in human cancer and also provide the first evidence that EZH2 directly regulates apoptotic process in cancer cells.

Cell Death and Differentiation (2010) 17, 801-810; doi:10.1038/cdd.2009.162; published online 6 November 2009

The E2F transcription factor family members play pivotal roles in regulating multiple cellular functions including proliferation, differentiation and apoptosis. ${ }^{1,2}$ Among them, E2F1 is the best-characterized owing to its unique feature to induce apoptosis. The pro-apoptotic activity of E2F1 can be mediated through both p53-dependent and independent pathways via target gene activation. These genes include ARF, ${ }^{3} \mathrm{TP}^{2},{ }^{4,5}$ APAF $-1^{6}$ and $\mathrm{BH} 3$ only BCL2 family members, including Bim, PUMA and Noxa. ${ }^{7,8}$ During tumorigenesis, it is believed that the E2F1 pro-apoptotic activity needs to be restrained to allow efficient transformation. While a role for E2F1 in apoptosis induction has been well established, the oncogenic signaling pathways restricting E2F1's pro-apoptotic potential in human cancer remain poorly defined.

It is well-known that activation of E2F1 can induce both proliferation and apoptosis. A compelling question is how E2F1-dependent apoptosis is inhibited in tumorigenesis to allow cancer cells to evade apoptosis. In addition to p53 deficiency as an important mechanism to cripple E2F1dependent apoptosis in human cancer, other potential oncogenic signals, including $\mathrm{PI} 3 \mathrm{~K},{ }^{9,10} \mathrm{MDM}^{11-13}$ and $\mathrm{HDAC}^{8}$ have also been reported to antagonize the apoptotic activity of E2F1 in cancer cells. As such, pharmacologic or biological agents that inhibit the above regulators have been shown to promote E2F1-dependent apoptosis in cancer cells. ${ }^{8,9,13,14}$

In addition to oncogenic lesions, aberrant epigenetic events leading to transcriptional inactivation of crucial tumor suppressors are also required for efficient malignant transformation. ${ }^{15,16}$ Accumulating evidence suggests that these two types of oncogenic lesions are often interconnected. For example, the oncogene Ras-mediated cellular transformation has been shown to be coupled with the polycomb proteinassociated epigenetic event that causes the inactivation of apoptotic Fas gene expression, thus inhibiting signals that would otherwise promote apoptosis. ${ }^{17}$ In addition, c-Mycdriven transformation in mammary epithelial cells can lead to the epigenetic silencing of natural inhibitors of Wnt signaling pathway, DKK1 and SFRP1, contributing to an aberrant activation of oncogenic $\beta$-catenin. ${ }^{18}$ These studies underscore the crucial role of epigenetic mechanisms in regulating oncogene-induced apoptosis during transformation.

Polycomb protein EZH2 is a histone methyltransferase that is frequently over-expressed in a wide variety of human malignancies. ${ }^{19,20} \mathrm{EZH} 2$ functions to suppress a number of tumor suppressor gene expression, such as E-cadherin, ${ }^{21}$

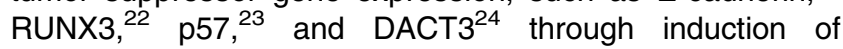
repressive histone methylation on $\mathrm{H} 3$ lysine 27 (H3K27Me3), thus promoting cell proliferation, metastasis, and invasion. ${ }^{20,23,25,26}$ Interestingly, EZH2 has been previously shown to be a downstream target of E2F1. ${ }^{27}$ This raises the possibility that an EZH2-mediated epigenetic event may have a functional link to E2F1-mediated cellular functions, such as apoptosis.

In this study, we show that the activation of EZH2 by E2F1 inhibits E2F1-mediated apoptosis in cancer cells and this is

\footnotetext{
${ }^{1}$ Cancer Biology and Pharmacology, Genome Institute of Singapore, $A^{*}$ Star (Agency for Science, Technology and Research), Biopolis, Singapore and ${ }^{2}$ Department of Physiology, Yong Loo Lin School of Medicine, National University of Singapore, Singapore, Singapore

*Corresponding author: Q Yu, Cancer Biology and Pharmacology, Genome Institute of Singapore, 60 Biopolis Street 0201, Sinagpore 138672, Sinagpore.

Tel: 657478 8127; Fax: 656478 9003; E-mail: yuq @ gis.a-star.edu.sg

Keywords: EZH2; E2F1; apoptosis; epigenetics; Bim

Abbreviations: APAF1, apoptosis protease-activating factor 1; ARF, alternative reading frame; BH3, BCL-2 homology 3; EZH2, enhancer of zeste homolog 2; HDAC, histone deacetylase; MDM2, human homolog of mouse double minute 2; MCL-1, myeloid leukemia cell differentiation protein 1; PI3K, phosphatidylinoditol-3-kinase; PRC2, polycomb repressive complex 2

Received 07.7.09; revised 17.9.09; accepted 18.9.09; Edited by J Silke; published online 6.11 .09
} 
achieved through epigenetic repression of Bim expression. Considering the frequent deregulation of both $\mathrm{E} 2 \mathrm{~F} 1$ and $\mathrm{EZH} 2$ in human cancer, the fail-safe mechanism we identified here provided a plausible mechanistic insight into the regulation of E2F1 apoptotic activity in cancer cells and also the role of $\mathrm{EZH} 2$ in promoting transformation.

\section{Results}

EZH2 suppresses E2F1-dependent Bim induction. To investigate the epigenetic process linked to the E2F associated transformation, we began to use the human lung fibroblast cells IMR90 and IMR90 cells transformed by oncoprotein E1A. One of the known mechanisms by which $E 1 A$ induces cellular transformation is through the inactivation of tumor suppressor $\mathrm{pRB}$, thereby leading to the activation of E2F. ${ }^{28}$ To understand the epigenetic factors potentially involved in E1A-induced transformation and E2F1 activation, we profiled the gene expression of known E2F1 targets and epigenetic regulators in IMR90 and IMR90/E1A cells. The data show the upregulation of many E2F1 target genes, including BCL2L11 (encoding Bim), E2F1, E2F2, CCNA1/2 and CCNE2 in IMR90/E1A cells compared to the IMR90 cells, confirming the activation of E2F1 in this transformation system (Figure 1a). Among several families of epigenetic regulators such as HDAC, DNMT and HMT we surveyed, histone methyltrasnferase EZH2 stood up as the most upregulated in this system, while others showed not much change or only modest induction (Figure 1b). Western blot analysis confirmed the array data and show that IMR90/E1A cells expressed a higher level of E2F1 and its downstream target genes, such as Cyclin A, p73 and Bim, either associated with E2F1-induced cell proliferation or apoptosis $^{29,30}$ (Figure 1c). EZH2 protein level was also verified to be upregulated in IMR90/E1A as compared to the IMR90 cells, and accordingly, the associated repressive histone mark trimethylated histone $\mathrm{H} 3$ lysine 27 (H3K27me3) was also elevated (Figure 1c). This result is in agreement with the previous report that identified $\mathrm{EZH} 2$ as an E2F1 target. $^{27}$

To confirm that the differential expression of the above E2F1 targets such as EZH2 and Bim in IMR90 and IMR90/E1A cells is truly E2F1-dependent, we depleted E2F1 expression using short interference RNA (siRNA)mediated gene silencing in IMR90/E1A cells. The results show that depletion of E2F1 efficiently ablated the protein expression of both EZH2 and Bim in IMR90/E1A cells (Figure 1d), further validating their E2F1 dependency in this system. Of notice, E2F1 knockdown also eliminated the expression of the other two activating E2F members E2F2 and E2F3 (Figure 1d), however, the induction of Bim expression is not E2F2 or E2F3 dependent, because depletion of E2F2 or E2F3 using specific siRNA respectively did not reduced Bim expression level (Supplementary Figure S1a). All the E2F1, 2, and 3 have been previously shown to regulate $E Z H 2{ }^{27}$ This finding explained the efficiency of E2F1 knockdown on EZH2 expression. In spite of the up-regulation of pro-apoptotic targets, IMR90/E1A cells grew faster than IMR90 cells. This suggests that the pro-apoptotic activity of
E2F1 in these cells must be restrained in order to support the proliferation and survival of these cells. The activation of EZH2H3K27me3 by E2F1 provides a hypothesis in which oncogenic transformation, induced by the $\mathrm{pRB} / \mathrm{E} 2 \mathrm{~F} 1$ pathway might be functionally linked to an EZH2-mediated epigenetic event.

To test the above hypothesis, we first examined whether siRNA-mediated knockdown of EZH2 has any effect on the expression of E2F1 pro-apoptotic targets. Thus, IMR90/E1A cells were transfected with EZH2-specific siRNA or a negative control siRNA (NC). RT-PCR analysis of a panel of wellknown E2F1 pro-apoptotic targets indicates that knockdown of $E Z H 2$ resulted in a marked increase in BIM mRNA expression, but had no obvious effect on other known E2F1 apoptotic targets, including TP73, NOXA, ARF, and APAF1 (Figure 1e), as well as other BCL2 family members (Supplementary Figure S1b). Western blot analysis confirmed the RT-PCR data and showed that EZH2 depletion led to a marked accumulation of Bim protein in a time-dependent manner (Figure 1f), but had no effect on p73 and Apaf1 (Figure 1g). EZH2, SUZ12 and EED are the three key components of the Polycomb repressor complex 2 (PRC2) and they depend on each other for the integrity of the protein complex. As shown in Figure 1g, EZH2 knockdown also depleted the SUZ12 and EED, indicating that EZH2 knockdown is sufficient to disrupt the entire PRC2 complex in these cells which is consistent with the previous report. ${ }^{27}$ To confirm the specific effect of EZH2 knockdown, we conducted the rescue experiment by cotransfection of $E Z H 2$ plasmid with another EZH2 siRNA that targets the $5^{\prime}-U T R$ of EZH2 mRNA and thus is unable to affect the ectopic expression of exogenous wild-type EZH2. The data in Figure $1 \mathrm{~h}$ show that this EZH2 siRNA also induced Bim expression and the cotransfection with an $\mathrm{EZH} 2$ expressing plasmid abrogated the Bim induction. These experiments demonstrate that inactivation of endogenous EZH2 complex leads to selective induction of E2F1 pro-apoptotic target Bim.

We next tested whether the ectopic expression of EZH2 is sufficient to inhibit Bim expression. IMR90/E1A cells were transfected with a DNA plasmid expressing EZH2 or EZH2 that lacks the SET domain required for the catalytic activity. As shown in Figure 1i, cells expressing ectopic EZH2 exhibited a lower level of Bim expression compared to the vector control. By contrast, the mutant EZH2 had no such an inhibitory effect on Bim expression (Figure 1i). Together with the previous data obtained using siRNA-mediated EZH2 knockdown, we conclude that $\mathrm{EZH} 2$ suppresses the expression of Bim. To extend our observations, we also knocked down EZH2 in human osteosarcoma Saos-2 and U2OS cells, prostate cancer PC3 and breast cancer MCF-7 cells, and observed Bim upregulation in these cells (Supplementary Figure S1c), therefore suggesting that $\mathrm{EZH} 2$-mediated Bim repression also extends to other cancer cell lines.

Upregulation of Bim upon removal of EZH2 is E2F1dependent. To demonstrate that the Bim induction upon EZH2 knockdown described above is E2F1-dependent, we tested whether the concomitant knockdown of E2F1 and EZH2 can reverse Bim induction. Thus, IMR90/E1A cells were transfected with EZH2 siRNA, E2F1 siRNA or both. The results show that the $\mathrm{EZH} 2$ siRNA-induced Bim increase was 
E2F1 targets

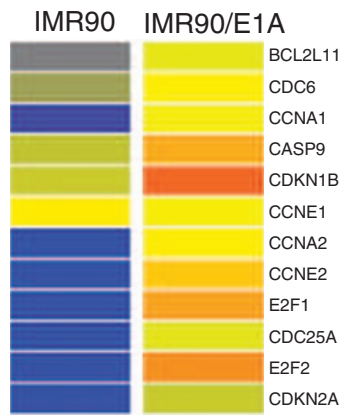

b Epigenetic regulators

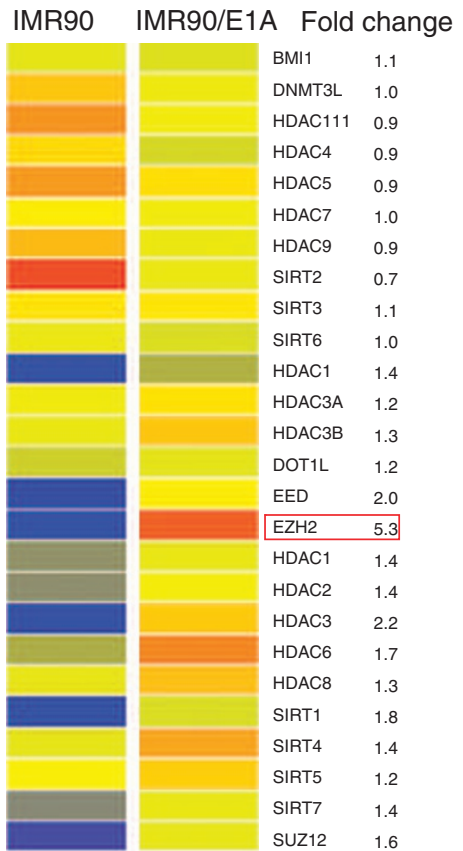

C

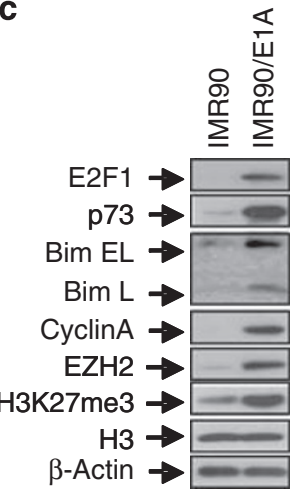

d

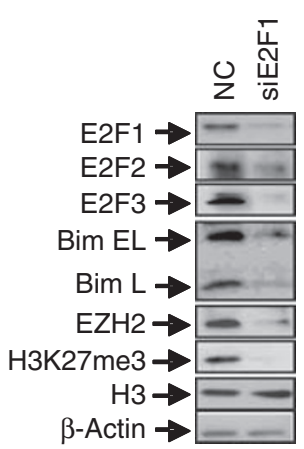

e

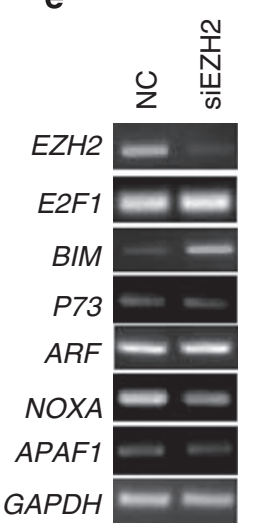

f

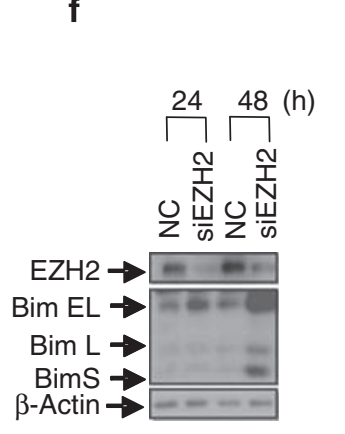

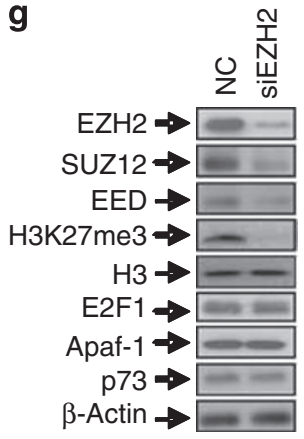
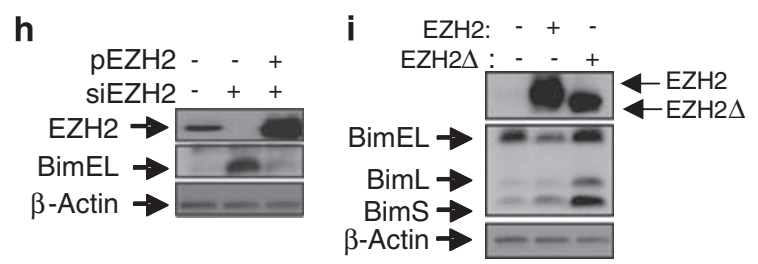

Figure 1 EZH2 represses E2F1-induced Bim expression. (a) Gene clustering showing the activation of E2F1 target genes in IMR90/E1A cells. (b) Gene clustering showing the expression profile of epigenetic regulators in IMR90 and IMR90/E1A cells. (c) IMR90/E1A cells express higher level of E2F1 and EZH2 than that in IMR90 cells. Western blot analysis showing the expression of E2F1, p73, Bim, cylinA, EZH2 and related H3K27me3. $\beta$-actin is used as a loading control. (d) The expression of Bim and EZH2 is E2F1-dependent. IMR90/E1A cells were transfected with negative control siRNA (NC) or E2F1 siRNA for 48 h, the expression of Bim, E2F1, E2F2, E2F3, EZH2 and related H3K27me3 were analyzed by Western blot analysis. (e) EZH2 depletion induces Bim transcription. IMR90/E1A cells were treated with NC or EZH2 siRNA for $48 \mathrm{~h}$, and mRNA levels of BIM, EZH2, ARF, E2F1 APAF1, NOXA and P73 were detected by RT-PCR. GAPDH was used as a loading control. (f) IMR90/E1A cells were transfected with EZH2 siRNA for 24 and $48 \mathrm{~h}$, and its effect on Bim protein level was shown by Western blot. (g) Cells were treated as above. The effects of EZH2 knockdown on other indicated E2F1 target genes and SUZ12 and EED are shown by Western blot analysis. (h) IMR90/E1A cells were transfected with a different siRNA targeting $5^{\prime}$-UTR of EZH2 alone or together with PCDNA4-EZH2 expression vector and the change of EZH2 and Bim were analyzed by Western blot analysis. (i) IMR90/E1A cells were transfected with wild-type EZH2 or EZH2Ä, which lacks the SET domain, the effect on Bim was assessed by Western blot analysis

abrogated by concomitant knockdown of E2F1, indicating that $\mathrm{EZH} 2$ depletion upregulating Bim expression in an E2F1-dependent fashion (Figure 2a). To further demonstrate this regulation in cancer cells, we performed the above experiments in human lung carcinoma cell lines A549 and H1299. In both cancer cell lines, knockdown of EZH2 resulted in increased Bim expression, which was completely reversed by double-knockdown of EZH2 and E2F1 (Figure 2b). Hence, in both transformed and tumorderived cell lines, we demonstrated that EZH2 knockdown promotes E2F1-dependent Bim expression.
The tumor suppressor $\mathrm{pRB}$ is a negative regulator of E2F1 activity $^{31}$ and is wild-type in $\mathrm{H} 1299$ cells. $^{32}$ Therefore, we examined the effect of pRB on E2F1-mediated Bim expression and how it may be regulated by EZH2. H1299 cells were transfected with siRNAs targeting $\mathrm{pRB}, \mathrm{EZH} 2$, or both siRNAs. The result shows that $\mathrm{pRB}$ depletion led to a clear induction of E2F1 as expected, but only a moderate up-regulation of Bim (Figure 2c). Double-knockdown of pRB and $\mathrm{EZH} 2$ caused a synergistic induction of Bim expression as compared to the knockdown of $\mathrm{pRB}$ or $\mathrm{EZH} 2$ alone (Figure 2c). These results further show that EZH2 inhibits 
a

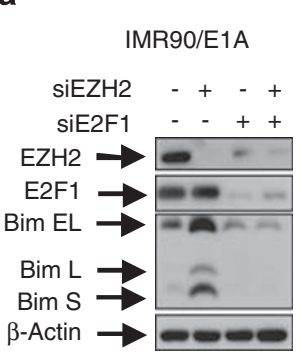

b

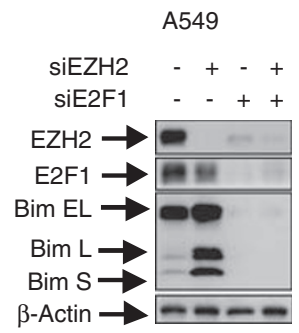

C

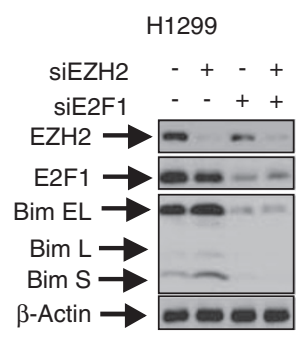

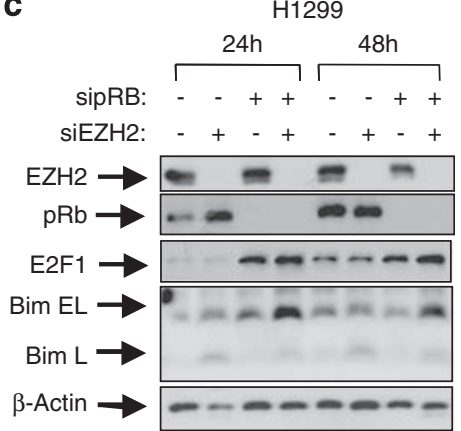

Figure 2 Upregulation of Bim upon removal of EZH2 is E2F1-dependent. (a) IMR90/E1A cells were treated with negative control (NC) siRNA, EZH2 siRNA, E2F1 siRNA or both for $48 \mathrm{~h}$ and the cells were harvested for Western blot analysis of EZH2, E2F1 and Bim expression. (b) Human lung carcinoma cell lines $\mathrm{H} 1299$ and A549 were treated with NC siRNA, EZH2 siRNA, E2F1 siRNA or both EZH2 and E2F1 siRNAs for $48 \mathrm{~h}$ and the cells were harvested for Western blot analysis of EZH2, E2F1 and Bim expression. (c) H1299 cells were treated with NC siRNA, EZH2 siRNA, pRBsiRNA or both pRB and EZH2 siRNAs for 24 and $48 \mathrm{~h}$. Cells were harvested and EZH2, pRB, E2F1 and Bim protein levels were analyzed by Western blotting

E2F1-mediated Bim expression and the removal of EZH2 promotes E2F1-dependent Bim expression.

EZH2 directly binds to the BIM promoter. To determine if $B I M$ is a direct target of EZH2, we performed chromatin immunopreciptation (ChIP) assays in both IMR90/E1A and $\mathrm{H} 1299$ cells to investigate whether EZH2 binds to the BIM gene locus. The chromatin precipitated from EZH2 antibody was analyzed by quantitative PCR using pairs of primers spanning around a $5.0 \mathrm{~kb}$ region surrounding the transcription start site (TSS). The results obtained from both cell lines identified EZH2 bound to BIM locus at about $500 \mathrm{bp}$ upstream and $800 \mathrm{bp}$ downstream of the TSS. Corresponding to the EZH2 enrichment, we detected a similar enrichment pattern of EZH2 associated H3K27me3 (Figure $3 \mathrm{a}$ ). EZH2 binding to BIM was specific since a negative control IgG failed to do so. The detection of EZH2 and H3K27me3 around $\pm 1.0 \mathrm{~kb}$ of TSS is consistent with the recent large scale mapping of and EZH2 H3K27me3 enrichment sites in human genome. ${ }^{33,34}$ Furthermore, we did not detect the binding of EZH2 to $P 73$ (Figure 3b), which is in agreement with the previous finding that EZH2 does not regulate $P 73$ expression (see Figure 1g). The levels of enrichment of EZH2 and H3K27me3 at BIM are comparable to that in the CDKN1C gene, a known EZH2 target in cancer cells (Figure $3 \mathrm{c}$ ), ${ }^{23}$ validating the specificity of the ChIP assay. In addition, we also detected the binding of EZH2 to BIM in U2OS and MCF-7 cells (Supplementary Figure S2). Knockdown of EZH2 clearly abrogated the EZH2 recruitment to the Bim locus (Figure 3d, left panel), while it did not affect E2F1 binding to a previously identified E2F1 binding site at $B I M^{\beta}$ (Figure 3d, right panel). By contrast, knockdown of E2F1 ablated both the E2F1 and EZH2 recruitment to BIM. The result confirms EZH2 as a downstream component of the E2F1 pathway. Taken together, these results show that $B I M$ is a direct target of EZH2 and that EZH2 binding to BIM is regulated by E2F1.

EZH2 regulates ectopic E2F1-induced apoptosis. To directly demonstrate that EZH2 affects E2F1-dependent apoptosis, we used a p53-deficient stable Saos-2 cell line containing an ER-E2F1 fusion protein (E2F1 fused to the hormone binding domain of human estrogen receptor) that is responsive to 4 -hydroxytamoxifen $(\mathrm{OHT}){ }^{35}$ In this system, activation of ectopic E2F1 induces apoptosis. Induction of both EZH2 and Bim by E2F1 was confirmed in this system (Figure 4a), and EZH2 knockdown promoted E2F1dependent Bim induction (Figure 4b). Activation of ERE2F1 by OHT triggered much more robust apoptosis when cells were depleted of EZH2 (68 versus $27 \%$ ), which was not seen in the control cells that did not express ER-E2F1 (25 versus 29\%) (Figure 4c). Similar results were also observed in p53-wild type stable U2OS/E-E2F1 cell line (Supplementary Figure S3). These results further indicate that E2F1-dependent apoptosis is partially neutralized by a concomitant EZH2 induction, which is independent of p53 status. Again, double-knockdown of EZH2 and Bim blunted the pro-apoptotic effect of EZH2 siRNA in the presence of OHT (Figure 4c), validating the essential role of Bim in this apoptosis.

EZH2 regulates E2F1-dependent apoptosis triggered by protein kinase inhibitors. Having shown that $\mathrm{EZH} 2$ regulates E2F1-dependent Bim expression, we next determined whether this modulation contributes to E2F1's apoptotic activity. EZH2 knockdown results in either growth arrest (senescence) or apoptosis. ${ }^{27,36}$ We found that in both IMR90/E1A and H1299 cells, knockdown of EZH2 induced a strong growth arrest instead of apoptosis. This suggests that the Bim induction following EZH2 depletion is insufficient to induce apoptosis in these cells, indicating that the proapoptotic potential of E2F1 remains inhibited due to additional control factors. It has been previously reported that the oncogenic kinase signaling PI3K/AKT pathway counteracts E2F1-dependent apoptosis, and as such, a small molecule PI3 K inhibitor can efficiently switch on E2F1dependent apoptosis. ${ }^{9,10}$ We therefore hypothesized that E2F1-dependent apoptosis in transformed cells might be also inhibited by certain protein kinase signaling, in addition to EZH2.

To identify small molecule protein kinase inhibitors that might activate E2F1-dependent apoptosis, we therefore 
a
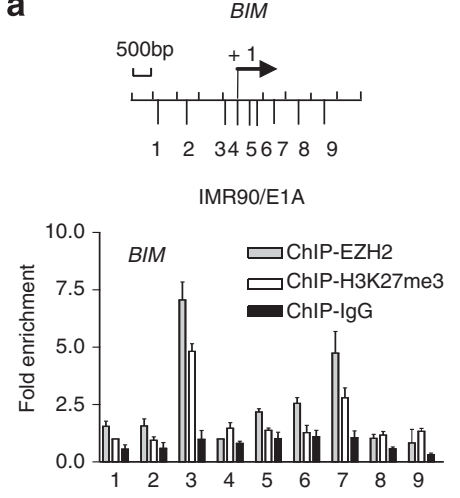

$\mathrm{H} 1299$
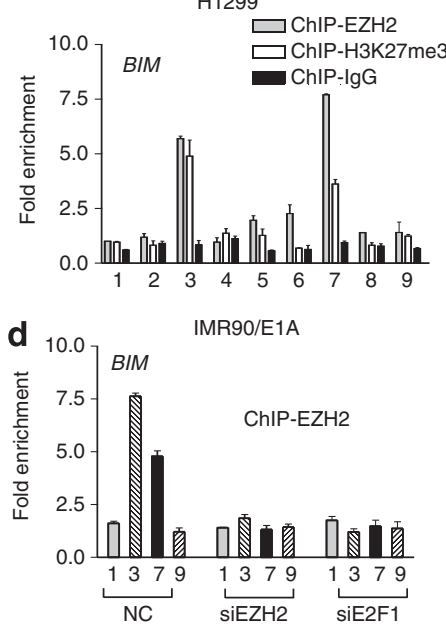

b

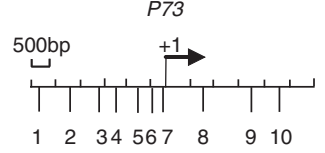

IMR90/E1A

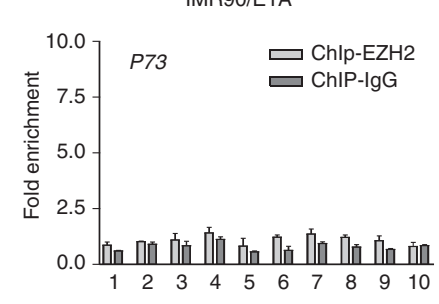

H1299
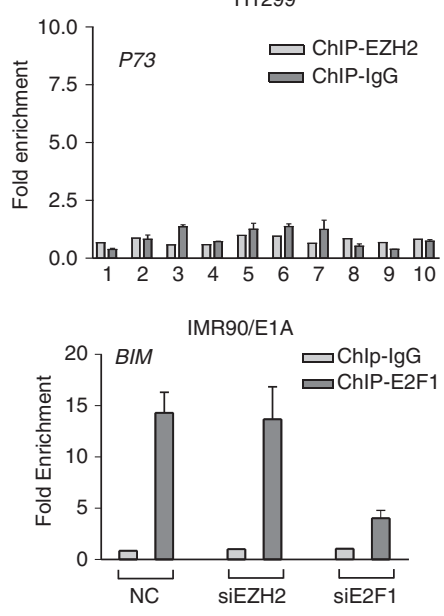

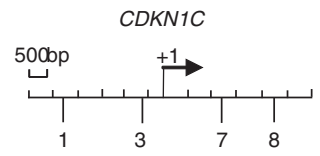

H1299

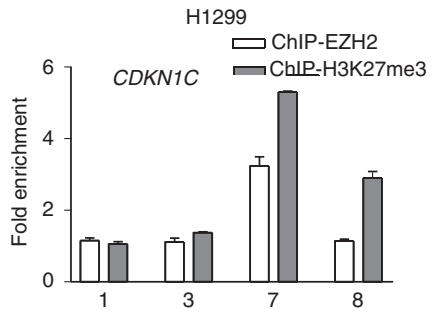

Figure $3 \mathrm{EZH} 2$ binds to the BIM promoter. (a) ChIP assays were performed in IMR90/E1A and H1299 cells using antibodies against EZH2, H3K27me3 or a negative control IgG and analyzed by quantitative PCR. Genomic DNA fragments covering $\sim 5.0 \mathrm{~kb}$ region surrounding the transcription start site (TSS) of BIM locus for PCR analysis were indicated with numbers. The relative enrichments (bound/input) (mean \pm S.D. of triplicate measurement) encompassing the indicated regions are shown at $B I M$ or $P 73$ locus. (b) The same analysis for P73 locus as in (a). (c) A known EZH2-H3K27me3 target CDKN1C is shown here as a positive control for ChIP assay. (d) IMR90/E1A cells were treated with negative control (NC) siRNA, EZH2 siRNA or E2F1 siRNA and the ChIP assays were conducted using antibody against EZH2 (left) or E2F1 (right). ChIPPCR was performed to detect the EZH2 binding to Bim (left). The effect of EZH2 or E2F1 knockdown on E2F1 binding to BIM, using a previously identified E2F1 binding site at the BIM locus is shown on the right. Shown are the relative enrichments (bound/input) in mean \pm S.D. of triplicate measurement

conducted a screen of 70 small molecule protein kinase inhibitors in IMR90 and IMR90/E1A cells. We identified 8 kinase inhibitors that seemed to induce significantly more apoptosis in IMR90/E1A cells as compared to IMR90 cells, suggesting that these kinase inhibitors may have an effect on the RB/E2F1 pathway. Among them, 6 kinase inhibitors showed the ability to trigger further enhanced apoptosis in IMR90/E1A cells when EZH2 was depleted (Supplementary Figure S4). D9 (Ro-318220) and F1 (5-lodostinbercidin), two kinase inhibitors that are reported to mainly target protein kinase $\mathrm{C}^{37}$ or adenosine kinase, ${ }^{38}$ respectively, produced the most robust apoptosis in response to EZH2 knockdown and thus were chosen for further study. As shown in Figure $5 \mathrm{a}$, treatment of IMR90/E1A cells with these two kinase inhibitors dramatically increased apoptosis upon EZH2 knock down. Since such an effect was not observed in the parental cells IMR90, the increased apoptosis is believed to be pRB/E2F1related (Figure $5 \mathrm{a}$ ). Importantly, this effect was not limited to IMR90/E1A cells, but was also observed in cancer cell line H1299 and A549 (Figure 5b). Of note, we did not detect an obvious effect of PI3 K inhibitor in our system, indicating that regulation of $\mathrm{PI} 3 \mathrm{~K}$ signaling on $\mathrm{E} 2 \mathrm{~F} 1$-dependent apoptosis as

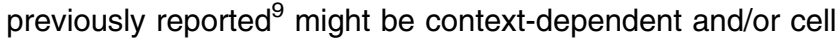
type specific.

The apoptotic effect after EZH2 knockdown was further confirmed by using EZH2 siRNA targeting $5^{\prime}$-UTR in a rescue experiment. Cotransfection of $\mathrm{EZH} 2$ plasmid with $\mathrm{EZH} 2$ siRNA abrogated the increased apoptosis induced by D9 or $\mathrm{F} 1$ upon $\mathrm{EZH} 2$ depletion (Figure $5 \mathrm{c}$ ), excluding the possibility of an off-target effect of EZH2 siRNA.

We next validated whether the strong apoptosis described above is dependent on E2F1 and Bim. The experiments performed in both IMR90/E1A and H1299 cells showed that $\mathrm{EZH} 2$ depletion-induced apoptotic response to D9 or F1 was largely abrogated when these cells were concomitantly transfected with E2F1 or Bim siRNA (Figure 5d and Supplementary Figure S5). These findings confirmed that the increased apoptosis to D9 and F1 treatment following $\mathrm{EZH} 2$ depletion at least in large part depends on E2F1-Bim pathway. Taken together, these results demonstrate that 


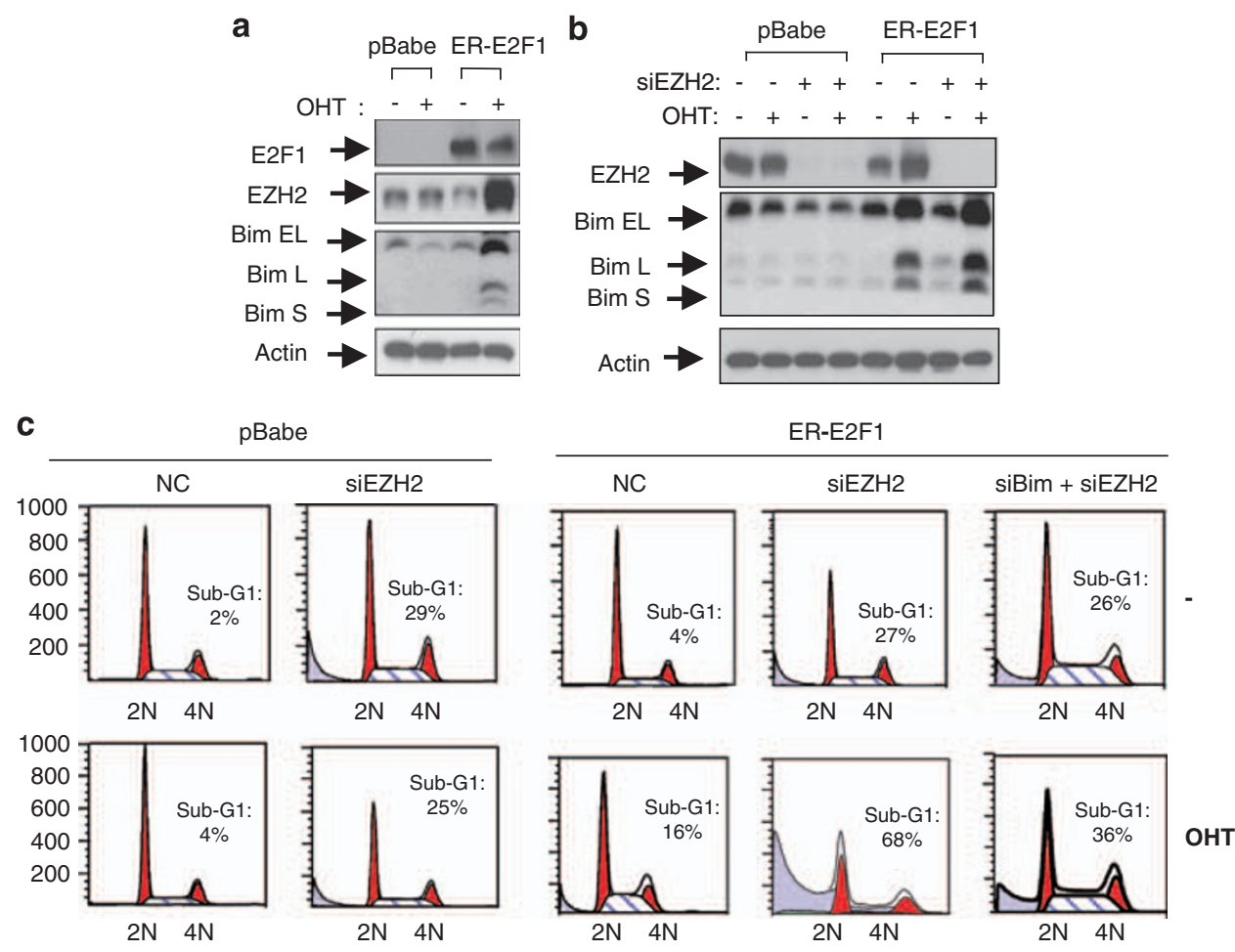

Figure 4 EZH2 regulates ectopic E2F1-induced apoptosis. (a) Western blot analysis of EZH2 and Bim in Saos-2 cells expressing the control vector (pBabe) or ER-E2F1. Cells were left untreated or treated with $300 \mathrm{nM} 4$-hydroxytamoxifen $(\mathrm{OHT})$ for $24 \mathrm{~h}$ and Western blot were performed to analyze the protein levels of E2F1, EZH2 and Bim. (b) EZH2 knockdown promotes the E2F1-induced up-regulation of Bim in Saos-2 cells expressing ER-E2F1. Saos-2 cells expressing pBabe or ER-E2F1 were transfected with negative control siRNA (NC) or EZH2 siRNA in the presence or absence of OHT for $8 \mathrm{~h}$, cells were harvested to assess the expression levels of EZH2 and Bim. (c) EZH2 depletion promoted E2F1-depependent apoptosis. Cells were treated as above for $48 \mathrm{~h}$ and apoptosis was analyzed using PI staining and FACS analysis. To validate the role of Bim, double knock down of Bim and EZH2 was performed in absence or the presence of OHT. The apoptosis was measured by PI staining and FACS analysis. The numbers of apoptotic cells in Sub-G1 are indicated

E2F1-dependent apoptosis in certain cellular context is protected by EZH2. EZH2 depletion may alter the balance of pro-and anti-apoptotic factors, priming the cell death when conditions are appropriate. Since E2F1 can induce apoptosis through either p53-dependent or p53-independent mechanisms, the fact that p53 is deficient in $\mathrm{H} 1299$ cells further indicates that E2F1-mediated apoptosis enhanced by EZH2 depletion does not depend on p53.

Downregulation of MCL-1 by protein kinase inhibitors synergizes with Bim induction upon EZH2 knockdown to cause Bax activation and apoptosis. We next investigated the potential mechanism by which EZH2 knockdown synergizes potently with the two protein kinase inhibitors D9 and F1-induced apoptosis. Because the synergistic apoptosis describe above is Bim-dependent, we reasoned that the synergy may route through the intrinsic apoptosis pathway through the coordination between Bim and the other members of Bcl-2 family. We thus analyzed the changes of Bcl-2 family members in drug treated cells with or without the $\mathrm{EZH} 2$ knockdown. To avoid the late secondary effects resulting from the massive apoptosis, we analyzed the early molecular events leading to apoptosis following the drug treatment. Pro-and anti-apoptotic members of $\mathrm{Bcl}-2$ family, including $\mathrm{MCL}-1, \mathrm{BCl} 2, \mathrm{BCL}-\mathrm{XL}, \mathrm{BCL}-\mathrm{Xs}$, Bax, caspase 3 and PARP were analyzed by western blotting. Strikingly, D9 and F1 treatment for $4 \mathrm{~h}$ induced dramatic downregulation of MCL-1, without affecting other $\mathrm{Bcl}-2$ members (Figure 6a). This change was not sufficient to induce caspase 3 and PARP cleavage; but in cells transfected with EZH2 siRNA thus expressing a higher level of Bim, the same treatment resulted in strong cleavage of both caspase 3 and PARP (Figure 6a), indicating the activation of intrinsic apoptotic process. FACS analysis using a Bax antibody that recognizes the conformationally active form of Bax indicates the Bax activation by D9 and F1 only in $\mathrm{EZH} 2$ siRNA-treated cells, even though the Bax protein levels remain unchanged (Figure $6 b$ ). MCL-1 is well known to inhibit apoptosis by binding and neutralizing anti-apoptotic Bim and Bax. The results suggest that neither Bim induction by $\mathrm{EZH} 2$ knockdown nor MCL-1 downregulation by $\mathrm{D} 9$ and $\mathrm{F} 1$ alone is sufficient to trigger the activation of the intrinsic apoptotic pathway under this condition, but the combination of the two processes does. Furthermore, further knockdown of Bim in EZH2-depleted cells (double knockdown) abrogated the cleavage of caspase 3 and PARP induced by D9 and F1 (Figure 6c), further substantiating the crucial role of $\mathrm{Bim}$ in promoting this apoptosis. Thus, these findings support a model in which Bim induction by $\mathrm{EZH} 2$ knockdown coordinates with MCL-1 


$$
\text { a }
$$

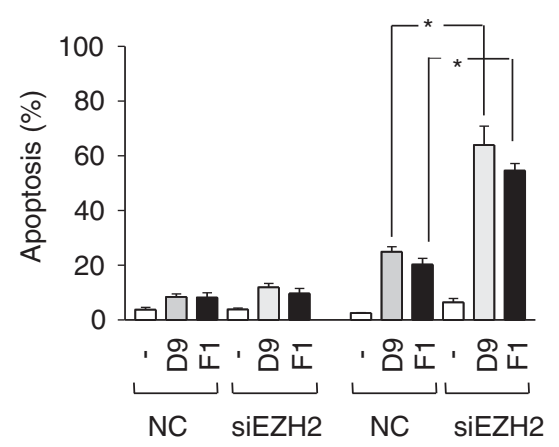

C

IMR90/E1A

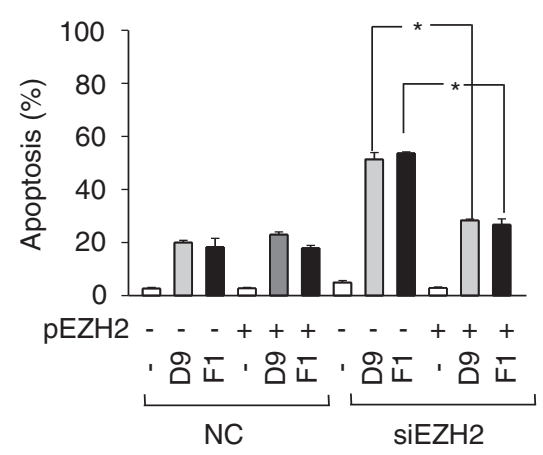

b

$\mathrm{H} 1299$

A549

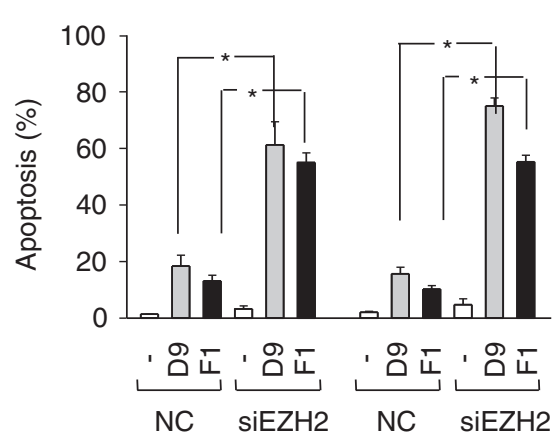

d 100

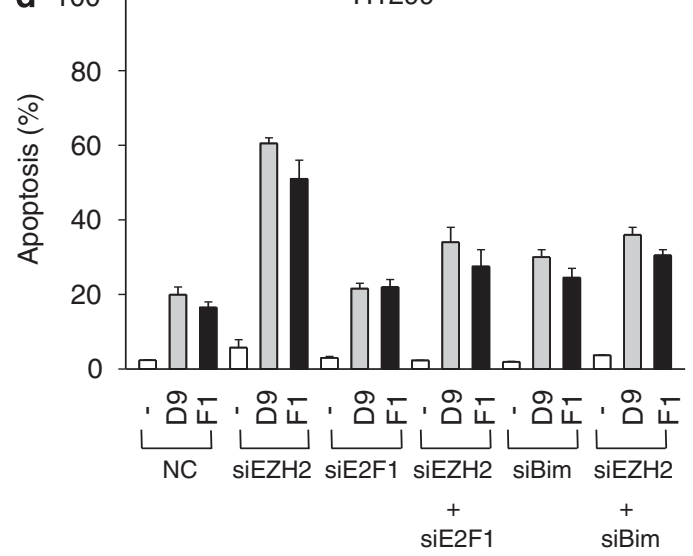

Figure 5 EZH2 regulates E2F1-dependent apoptosis triggered by kinase inhibitors. (a) IMR90 and IMR90/E1A cells were transfected with negative control (NC) siRNA or EZH2 siRNA followed by treatment with kinase inhibitor D9 (Ro 31-8220) or F1 (5-lodotubercidin). at $5 \mu \mathrm{M}$ for $24 \mathrm{~h}$. Cells were harvested for FACS analysis using PI staining and FACS analysis. Cell populations with sub-G1 content were defined as apoptotic cells, and data were represented as means \pm S.D. from three independent experiments. ${ }^{*} P<0.05$. (b) $\mathrm{H} 1299$ and A549 cells were transfected with NC or EZH2 siRNA followed by treatment with kinase inhibitor D9 (Ro 31-8220) or F1 (5-lodotubercidin) at $5 \mu \mathrm{M}$ for $48 \mathrm{~h}$. The apoptosis was determined by PI staining and FACS analysis. Means of three independent experiments were shown with standard deviations. ${ }^{*} P<0.05$. (c) Ectopic expression of EZH2 rescues EZH2 siRNA-induced apoptosis in response to D9 or F1 treatment. IMR90/E1A cells were co-transfected with EZH2 siRNA targeting the $5^{\prime} \mathrm{UTR}$ of EZH2 and an EZH2 expressing plasmid. The apoptosis was determined as in A. Means of three independent experiments were shown with standard deviations. (d) H1299 cells were treated with NC siRNA, EZH2 siRNA, E2F1 siRNA, Bim siRNA or both EZH2 and E2F1 siRNAs, or EZH2 and Bim siRNAs, followed by D9 or F1 for $48 \mathrm{~h}$. Cells were harvested for apoptosis analysis using PI staining and FACS analysis. Means of three independent experiments were shown with standard deviations

downregulation by $\mathrm{D} 9$ and $\mathrm{F} 1$ to induce synergistic Bax activation and apoptosis (Figure 6d).

\section{Discussion}

E2F1-dependent apoptosis has been the subject of intensive investigation due to the frequent deregulation of RB/E2F1 pathway in human malignancy. However, little is known about the oncogenic events regulating E2F1's apoptotic activity in cancer cells, which is believed to be critical for efficient malignant transformation by which allows cancer cells to evade apoptosis. Our findings define a novel regulatory mechanism that restrains E2F1 pro-apoptotic activity in human cancer which suggests that therapeutic inhibition of EZH2 may restore E2F1 apoptotic activity, thus providing a new treatment option for cancer.

In mammalian cells, E2F1 is equipped with the ability to induce both proliferation and apoptosis. It remains to be determined how this paradoxical function of E2F1 is balanced to prevent the onset of apoptotic activity of E2F1 during tumorigenesis. This process may involve multiple mechanisms. In addition to p53 deficiency that may alleviate E2F1-dependent apoptosis in cancer cells, oncogenic protein kinase signaling such as $\mathrm{PI} 3 \mathrm{~K}$ may also restrains E2F1-dependent apoptosis. ${ }^{9,10}$ Our current results provide evidence that polycomb protein $\mathrm{EZH} 2$, in collaboration with certain protein kinase signaling pathways, may restrain E2F1-dependent apoptosis in transformed cells.

Bim has been previously shown to be an apoptotic target of $\mathrm{E} 2 \mathrm{~F} 1 .{ }^{8}$ Our data show that $\mathrm{EZH} 2$ and associated H3K27me3 are enriched in the Bim promoter to suppress the Bim expression. As a component of PRC2 complex, EZH2 knockdown also resulted in the disruption of this entire repressor protein complex and this expected to effectively remove H3K27me3 for gene activation. Thus, the PRC2 occupancy of Bim promoter might be an important mechanism to restrict Bim induction by E2F1 or other factors in the cancer cells.

Through a screen of small molecule kinase inhibitors library, we identified several kinase inhibitors that promote 


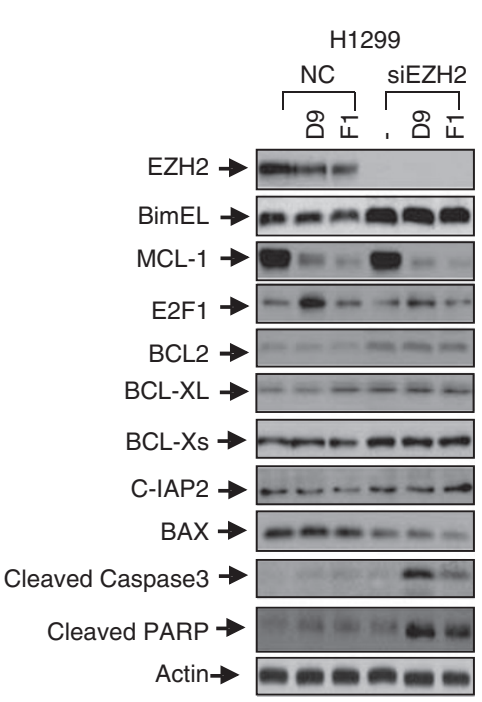

b

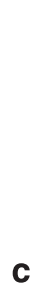

c

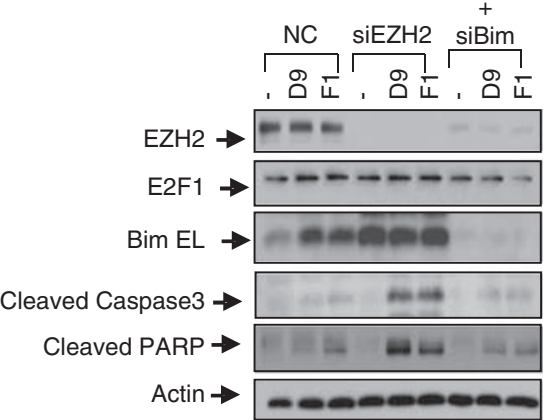

d

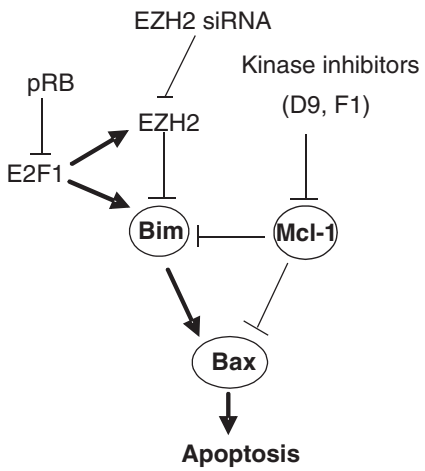

Figure 6 Downregulation of MCL-1 coordinates with Bim induction to mediate Kinase inhibitor-induced apoptosis. (a) Western blot analysis of Bcl-2 family members and E2F1, EZH2, caspase 3 and PARP cleavages in response to D9 and F1 for $4 \mathrm{~h}$ in H1299 1299 Hcells treated with NC or EZH2 siRNA. (b) H1299 cells treated as above were stained with monoclonal Bax antibody (6A7) that only recognizes the conformationally active Bax before performing analysis using FACS. (c) Western blot analysis of EZH2, E2F1, Bim, caspase 3 and PARP cleavage in cells treated with D9 and F1 upon knockdown of EZH2 alone or together with Bim. (d) Proposed model showing that knockdown of EZH2 cooperates with kinase inhibitors to induce Bax activiation and apoptosis through inducing Bim expression and downregulation of MCL-1, respectively

E2F1-dependent apoptosis together with EZH2 knockdown. In these cells, depletion of EZH2 to induce Bim alone was insufficient to induce obvious apoptosis, but it greatly enhanced apoptosis triggered by certain kinase inhibitors in E2F1-dependent manner. Mechanistically, we have demonstrated that the two kinase inhibitors D9 and F1 can cause a rapid downregulation of $\mathrm{MCL}-1$ protein, which coordinates with the induction of Bim by $\mathrm{EZH} 2$ knockdown to trigger a strong apoptosis through synergistic activation of Bax. To extend this observation, our data also suggest that Bim induction by $\mathrm{EZH} 2$ knockdown may also sensitize other apoptotic signals that rout through intrinsic apoptotic process. Thus targeting EZH2, which is often overexpressed in cancer, might be a useful approach to sensitize anti-cancer therapy or overcome drug resistance. Although this study is not aimed at identifying the exact kinase signaling that regulates E2F1 apoptosis, future work is required to achieve this based on the results presented here.

In addition, the data offers direct evidence that removal of EZH2 promotes E2F1-dependent apoptosis via ectopic E2F1 and thus establishes a crucial role of EZH2 in regulating E2F1 pro-apoptotic activity in cancer cells. Our data thus define a functional network between E2F1, EZH2 and Bim. This repressive effect of $\mathrm{EZH} 2$ on Bim appears to be selective as it does not exert much effect on other E2F1 pro-apoptotic targets. In this E2F1-dependent context, induction of EZH2 and thus repressing Bim expression constitutes a cellular circuitry that attenuates E2F1-dependent apoptosis, allowing the transformation to be efficient. Currently, the molecular pathways leading to $\mathrm{EZH} 2$ overexpression in human cancer is largely unknown. In addition to being a target of E2F1, EZH2 is also upregulated by Myc indirectly in B-cell lymphoma through an miRNA-mediated mechanism. ${ }^{39}$ Thus our findings that $\mathrm{EZH} 2$ inhibits Bim expression may suggest that
EZH2-mediated epigenetic events and oncogene signaling may be broadly interconnected through Bim. This connection may provide a molecular explanation for the restrained apoptotic activity of E2F1 in tumors cells. Indeed, it has been previously shown that Bim functions in vivo to regulate oncogene-induced tumorigenesis and the abrogation of Bim greatly facilitates Myc-induced tumor formation. ${ }^{40}$ Of particular interest is that Bim, unlike PUMA and Noxa, is not a p53 target and mediates p53-independent apoptosis. ${ }^{41}$ Hence, $\mathrm{EZH} 2$ suppression of Bim expression in the context of either E2F1 or Myc deregulation might be a scenario relevant for tumorigenesis regardless of p53 status.

Our data reveals a mechanistic link between $p R B / E 2 F 1$ pathway and EZH2-mediated epigenetic regulation, two important oncogenic pathways in human cancer. This regulation is reminiscent of the recent report that oncogene Rasinduced transformation is linked to Ras-directed epigenetic inactivation of apoptotic Fas gene, which is required for maintenance of a fully transformed phenotype. ${ }^{17}$ Together with our findings, these results indicate that oncogeneinduced transformation and epigenetic event in cancer are interconnected molecular pathways leading to efficient transformation during tumorigenesis.

In conclusion, our study reveals a novel mechanism by which E2F1-mediated apoptosis is controlled in cancer. This is achieved at least in part through EZH2 and its ability to suppress E2F1-dependent Bim expression. In addition, the extent of apoptosis observed after EZH2 depletion in response to certain kinase inhibitors encourages the therapeutic targeting of $\mathrm{EZH} 2$ as an important modulator of apoptosis selectively for cancer cells. Importantly, it provides an attractive approach to exploit both oncogene and epigenetic addiction in cancer cells, leading to a potential for a therapeutic window. 


\section{Materials and Methods}

Cell culture and Drug treatment. IMR90, Saos-2, H1299, A549, MCF-7, U2OS, and PC3 cells were obtained from ATCC (Manassas, VA). IMR90/E1A cells were kindly provided by Dr Claudio Brancolini (University of Di Udine, Italy). For drug treatment, cells were treated with $5 \mu \mathrm{M}$ Ro 31-8220, or $5 \mu \mathrm{M}$ 5-lodotubercidin for 24 or $48 \mathrm{~h}$ before harvested for FACS and Western blot analysis. Kinase inhibitors used in this study was purchase from BioMol (Plymouth Meeting, PA). EZH2 wild type and set domain deletion plasmid have been described previously. ${ }^{27}$

Microarray gene expression analysis, semi-quantitative RT-PCR and Taqman Assay. Total RNA was isolated using Trizol (Invitrogen) and purified with the RNeasy Mini Kit (Qiagen). Reverse transcription was performed using an RNA Amplification kit (Ambion). The microarray hybridization was performed using the Illumina Gene Expression Sentrix BeadChip HumanRef-8 V2, and data analysis was performed using GeneSpring software from Agilent Technologies as described. ${ }^{24}$

FACS analysis. FACS analysis for cell cycle analysis was performed as previously described (39). To detect activate Bax in cells, cells were fixed with Cytofix/Cytoperm solution (BD Pharmingen), stained first with the anti- Bax 6A7 monoclonal antibody (BD Pharmingen) and then with polyclonal rabbit anti-mouse immunoglobulin FITC (DakoCytomation), followed by FACS analysis.

Antibodies and siRNAs. Anti-EZH2 (AC22), anti-Bim (4582) were purchased from Cell Signaling, anti-E2F1 (KH95), anti-cyclinA (BF-683) antiE2F2 (c-20), anti-E2F3 (c-18) and anti-RB1 (IF-8) obtained from Santa Cruz, anttrimethylated H3K27 (07-449), anti-H3 (06-599), anti-Apaf1(06-957) were purchased from Upstate. Antibodies against p73 and c-Myc were products of Oncogene and Roche respectively. Specific siRNA target EZH2 RB1, E2F1 and Bim mRNA was described previously. $8,27,42,43$ The non-targeting control siRNA was purchased from Dharmacon (Lafayett, CO). A separate siRNA targeting $5^{\prime}$ UTR region of EZH2 with the following sequence $5^{\prime}$-CGGTGGGACTCAGAAGGCA-3' was obtained from Sigma-Proligo.

RT-PCR and ChIP. RT-PCR and ChIP assays were performed as described previously. ${ }^{24}$ Sonicated extracts were precleared and incubated with antibodies specific to either EZH2 (Active motif, Carlsbad, CA), E2F1 (c-20, Santa Cruz) or IgG ctrl (sc-2027, Santa Cruz) at $4^{\circ} \mathrm{C}$ overnight on a $360^{\circ} \mathrm{C}$ rotator. The immunoprecipitated DNA was quantitated by real-time quantitative PCR using Applied Biosystems 7900HT Fast Real-Time PCR System (Applied Biosystems). The enrichment of specific genomic regions was assessed relative to the input DNA. Primer sequences of the RT-PCR and ChIP-PCR are listed in the Supplementary Table S1.

Statistical analysis. The student's test was done to assess the difference of cell death between various treatment with siRNA and kinase inhibitors. $P<0.05$ was defined as the statistic significance.

\section{Conflict of interest}

The authors declare no conflict of interest.

Acknowledgements. We thank Dr Kristian Helin (European Institute of Oncology, Milan) for the ER-E2F1 plasmids and Dr Claudio Brancolini for providing the IMR90/E1A cells. This work was supported by the Agency for Science, Technology and Research of Singapore.

1. laquinta PJ, Lees JA. Life and death decisions by the E2F transcription factors. Curr Opin Cell Biol 2007; 19: 649-657.

2. Polager S, Ginsberg D. E2F-at the crossroads of life and death. Trends Cell Biol 2008; 18: $528-535$.

3. Bates S, Phillips AC, Clark PA, Stott F, Peters G, Ludwig RL et al. p14ARF links the tumour suppressors RB and p53. Nature 1998; 395: 124-125.

4. Irwin M, Marin MC, Phillips AC, Seelan RS, Smith DI, Liu W et al. Role for the p53 homologue p73 in E2F-1-induced apoptosis. Nature 2000; 407: 645-648.

5. Stiewe T, Putzer BM. Role of the p53-homologue p73 in E2F1-induced apoptosis. Nat Genet 2000; 26: 464-469

6. Moroni MC, Hickman ES, Lazzerini Denchi E, Caprara G, Colli E, Cecconi F et al. Apaf-1 is a transcriptional target for E2F and p53. Nat Cell Biol 2001; 3: 552-558.
7. Hershko T, Ginsberg D. Up-regulation of Bcl-2 homology $3(\mathrm{BH} 3)$-only proteins by E2F1 mediates apoptosis. J Biol Chem 2004; 279: 8627-8634.

8. Zhao Y, Tan J, Zhuang L, Jiang X, Liu ET, Yu Q. Inhibitors of histone deacetylases target the $\mathrm{Rb}-\mathrm{E} 2 \mathrm{~F} 1$ pathway for apoptosis induction through activation of proapoptotic protein Bim. Proc Natl Acad Sci USA 2005; 102: 16090-16095

9. Hallstrom TC, Mori S, Nevins JR. An E2F1-Dependent Gene Expression Program that Determines the Balance between Proliferation and Cell Death. Cancer Cell 2008; 13: $11-22$.

10. Hallstrom TC, Nevins JR. Specificity in the activation and control of transcription factor E2F-dependent apoptosis. Proc Natl Acad Sci USA 2003; 100: 10848-10853

11. Loughran O, La Thangue NB. Apoptotic and growth-promoting activity of E2F modulated by MDM2. Mol Cell Biol 2000; 20: 2186-2197.

12. Kitagawa M, Aonuma M, Lee SH, Fukutake S, McCormick F. E2F-1 transcriptional activity is a critical determinant of Mdm2 antagonist-induced apoptosis in human tumor cell lines. Oncogene 2008; 27: 5303-5314.

13. Laurie NA, Donovan SL, Shih CS, Zhang J, Mills N, Fuller C et al. Inactivation of the p53 pathway in retinoblastoma. Nature 2006; 444: 61-66.

14. Johnson L, Shen A, Boyle L, Kunich J, Pandey K, Lemmon M et al. Selectively replicating adenoviruses targeting deregulated E2F activity are potent, systemic antitumor agents. Cancer Cell 2002; 1: 325-337.

15. Baylin SB, Ohm JE. Epigenetic gene silencing in cancer-a mechanism for early oncogenic pathway addiction? Nat Rev Cancer 2006; 6: 107-116.

16. Esteller M. Epigenetics provides a new generation of oncogenes and tumour-suppressor genes. Br J Cancer 2006; 94: 179-183.

17. Gazin C, Wajapeyee N, Gobeil S, Virbasius CM, Green MR. An elaborate pathway required for Ras-mediated epigenetic silencing. Nature 2007; 449: 1073-1077.

18. Cowling VH, D'Cruz CM, Chodosh LA, Cole MD. c-Myc transforms human mammary epithelial cells through repression of the Wnt inhibitors DKK1 and SFRP1. Mol Cell Biol 2007; 27: 5135-5146.

19. Cao $R$, Zhang $Y$. The functions of $E(Z) / E Z H 2$-mediated methylation of lysine 27 in histone H3. Curr Opin Genet Dev 2004; 14: 155-164.

20. Varambally S, Dhanasekaran SM, Zhou M, Barrette TR, Kumar-Sinha C, Sanda MG et al. The polycomb group protein EZH2 is involved in progression of prostate cancer. Nature 2002; 419: 624-629.

21. Cao Q, Yu J, Dhanasekaran SM, Kim JH, Mani RS, Tomlins SA et al. Repression of E-cadherin by the polycomb group protein EZH2 in cancer. Oncogene 2008; 27: 7274-7284

22. Fujii $S$, Ito $K$, Ito $Y$, Ochiai $A$. Enhancer of zeste homologue 2 (EZH2) downregulates RUNX3 by increasing histone $\mathrm{H} 3$ methylation. J Biol Chem 2008; 283 : 17324-17332.

23. Yang X, Karuturi RK, Sun F, Aau M, Yu K, Shao R et al. CDKN1C (p57) is a direct target of EZH2 and suppressed by multiple epigenetic mechanisms in breast cancer cells. PLoS ONE 2009; 4: e5011.

24. Jiang X, Tan J, Li J, Kivimae S, Yang X, Zhuang L et al. DACT3 is an epigenetic regulator of Wnt/beta-catenin signaling in colorectal cancer and is a therapeutic target of histone modifications. Cancer Cell 2008; 13: 529-541.

25. Kleer CG, Cao Q, Varambally S, Shen R, Ota I, Tomlins SA et al. EZH2 is a marker of aggressive breast cancer and promotes neoplastic transformation of breast epithelial cells. Proc Natl Acad Sci USA 2003; 100: 11606-11611.

26. Tan J, Yang X, Zhuang L, Jiang X, Chen W, Lee PL et al. Pharmacologic disruption of Polycomb-repressive complex 2-mediated gene repression selectively induces apoptosis in cancer cells. Genes Dev 2007; 21: 1050-1063.

27. Bracken AP, Pasini D, Capra M, Prosperini E, Colli E, Helin K. EZH2 is downstream of the pRB-E2F pathway, essential for proliferation and amplified in cancer. EMBO J 2003; 22: 5323-5335

28. Nahle Z, Polakoff J, Davuluri RV, McCurrach ME, Jacobson MD, Narita M et al. Direct coupling of the cell cycle and cell death machinery by E2F. Nat Cell Biol 2002; 4: 859-864.

29. Bracken AP, Ciro M, Cocito A, Helin K. E2F target genes: unraveling the biology. Trends Biochem Sci 2004; 29: 409-417.

30. Putzer BM. E2F1 death pathways as targets for cancer therapy. J Cell Mol Med 2007; 11: 239-251.

31. Burkhart DL, Sage J. Cellular mechanisms of tumour suppression by the retinoblastoma gene. Nat Rev Cancer 2008; 8: 671-682.

32. Kawabe S, Nishikawa T, Munshi A, Roth JA, Chada S, Meyn RE. Adenovirus-mediated mda-7 gene expression radiosensitizes non-small cell lung cancer cells via TP53independent mechanisms. Mol Ther 2002; 6: 637-644.

33. Zhao XD, Han X, Chew JL, Liu J, Chiu KP, Choo A et al. Whole-genome mapping of histone $\mathrm{H} 3 \mathrm{Lys} 4$ and 27 trimethylations reveals distinct genomic compartments in human embryonic stem cells. Cell Stem Cell 2007; 1: 286-298.

34. Pan G, Tian S, Nie J, Yang C, Ruotti V, Wei H et al. Whole-genome analysis of histone H3 lysine 4 and lysine 27 methylation in human embryonic stem cells. Cell Stem Cell 2007; 1: 299-312.

35. Vigo E, Muller H, Prosperini E, Hateboer G, Cartwright $\mathrm{P}$, Moroni MC et al. CDC25A phosphatase is a target of E2F and is required for efficient E2F-induced $\mathrm{S}$ phase. Mol Cell Biol 1999; 19: 6379-6395. 
36. Kondo Y, Shen L, Cheng AS, Ahmed S, Boumber Y, Charo C et al. Gene silencing in cancer by histone $\mathrm{H} 3$ lysine 27 trimethylation independent of promoter DNA methylation. Nat Genet 2008; 40: 741-750.

37. Davies SP, Reddy H, Caivano M, Cohen P. Specificity and mechanism of action of some commonly used protein kinase inhibitors. Biochem J 2000; 351 (Pt 1): 95-105.

38. Lynch 3rd JJ, Jarvis MF, Kowaluk EA. An adenosine kinase inhibitor attenuates tactile allodynia in a rat model of diabetic neuropathic pain. Eur J Pharmacol 1999; 364: 141-146.

39. Sander S, Bullinger L, Klapproth K, Fiedler K, Kestler HA, Barth TF et al. MYC stimulates EZH2 expression by repression of its negative regulator miR-26a. Blood 2008; 112 : 4202-4212.
40. Egle A, Harris AW, Bouillet $P$, Cory $\mathrm{S}$. Bim is a suppressor of Myc-induced mouse $B$ cell leukemia. Proc Natl Acad Sci USA 2004; 101: 6164-6169.

41. Villunger A, Michalak EM, Coultas L, Mullauer F, Bock G, Ausserlechner MJ et al. p53- and drug-induced apoptotic responses mediated by $\mathrm{BH} 3-$ only proteins puma and noxa. Science 2003; 302: 1036-1038.

42. Semizarov D, Kroeger P, Fesik S. siRNA-mediated gene silencing: a global genome view. Nucleic Acids Res 2004; 32: 3836-3845.

43. Tan J, Zhuang L, Jiang X, Yang KK, Karuturi KM, Yu Q. Apoptosis signal-regulating kinase 1 is a direct target of E2F1 and contributes to histone deacetylase inhibitor-induced apoptosis through positive feedback regulation of E2F1 apoptotic activity. J Biol Chem 2006; 281: 10508-10515.

Supplementary Information accompanies the paper on Cell Death and Differentiation website (http://www.nature.com/cdd) 